\title{
SPECTROSCOPY OF Be STARS IN NGC 330
}

\author{
PAOLO A. MAZZALI and F. PASIAN \\ Osservatorio Astronomico, Via G.B.Tiepolo, 11, Trieste, Italy \\ D.J. LENNON \\ Universitäts Sternwarte, München, Germany \\ P. BONIFACIO \\ SISSA, Trieste, Italy \\ and \\ V. CASTELLANI \\ Istituto di Astronomia, Universitá di Pisa, Italy
}

Medium resolution $(2 \AA / \mathrm{px})$ but high s/n spectra of approximately twenty of the brightest blue stars in the young open cluster NGC 330 in the SMC have been obtained with EFOSC1 on the ESO 3.6m telescope, and analyzed in order to determine the atmospheric parameters and the evolutionary status of the stars. LTE and NLTE model atmosphere calculations were used to determine the stellar parameters. The $T_{\text {eff }}$ values were derived from fits of the UV continua for all stars where these were available, using Robertson's (1974) $B$ and $V$ photometry to scale the Kurucz model fluxes for metallicity $Z=0.1 Z_{\odot}$. Luminosities of the sample stars lie in the range $4.0<\log \left(L_{*} / L_{\odot}\right)<5.0$ and spectral types between B0 and late-B.

We find that all but one of the stars in our sample with spectral types in the range $\mathrm{B} 0-\mathrm{B} 3 \mathrm{~V}-\mathrm{II}$ show emission in $\mathrm{H}_{\alpha}$, confirming previous findings based on low resolution spectroscopy (Feast 1972) and photometry (Grebel et al. 1992) that the incidence of Be stars in this metal-poor cluster is very high (60-70\%, Grebel et al. 1992). This is a peculiar feature requiring explanation. Stars are classified as Be if they show emission in $\mathrm{H}_{\alpha}$, but in some the emission is observed also in $\mathrm{H}_{\beta}, \mathrm{H}_{\gamma}$ and $\mathrm{H}_{\delta}$. The resolution is too small $(\sim 100 \mathrm{~km} / \mathrm{s})$ to resolve any $V / R$ components in the emission lines.

For the strongest Be stars, where emission was present even in the cores of $\mathrm{H}_{\gamma}$ and $\mathrm{H}_{\delta}$, we tried to determine $T_{\text {eff }}$ and $\log g$ by comparing the profiles of their Balmer lines with those of stars with less emission, and by fitting the wings of the lines, which were assumed to be unaffected by the Be emission. A comparison with evolutionary tracks cun be found in Lennon et al. 1993. We measured $v_{\text {rot }} \sin i$ values from the widths of the $\mathrm{H}_{\alpha}$ emission, when present, and the emission intensity from the equivalent width of the emission itself. The various parameters of the stars in our sample are summarized in Table 1. The stars are referred to by their Robertson (1974) number.

The $\mathrm{H}_{\alpha}$ intensity is strongly correlated with the value of $v_{\text {rot }} \sin i$. This is in some sense an unexpected result, since if the $\mathrm{H}_{\alpha}$ emission arises from 
TABLE I

Parameters for the stars in NGC 330.

\begin{tabular}{rcccccr}
\hline STAR & $\begin{array}{c}\mathrm{T}_{\text {eff }} \\
\mathrm{K}\end{array}$ & $\log g$ & $\log \left(L / L_{\odot}\right)$ & $\begin{array}{c}\text { Type } \\
\mathrm{EW}\left(\mathrm{H}_{\alpha}\right) \\
\AA\end{array}$ & & $\begin{array}{r}v \sin i \\
\mathrm{~km} / \mathrm{s}\end{array}$ \\
\hline $\mathrm{A} 01$ & $29000 \pm 1000$ & $4.25 \pm 0.20$ & 4.74 & B0.5 Ve & -0.4 & 125 \\
$\mathrm{~A} 02$ & $16000 \pm 1000$ & $2.50 \pm 0.20$ & 4.86 & B4 Iab/b & - & $<100$ \\
B04 & $25000 \pm 1000$ & $3.90 \pm 0.20$ & 4.23 & B1.5 IVe & -2.4 & 180 \\
B05 & $22000 \pm 1000$ & $3.50 \pm 0.25$ & 4.16 & B2 IIIe & -38.7 & 300 \\
B06 & $22000 \pm 1000$ & $3.50 \pm 0.30$ & 4.21 & B2 IIIe & -26.9 & 300 \\
B07 & $22000 \pm 3000$ & $3.70 \pm 0.30$ & 4.09 & B2 III/IVe & -1.6 & 160 \\
B11 & $12000 \pm 2000$ & $3.25 \pm 0.50$ & 3.56 & B7 II/III & - & $<100$ \\
B12 & $22000 \pm 2000$ & $3.40 \pm 0.30$ & 4.22 & B2 IIIe & -37.5 & 300 \\
B13 & $22000 \pm 2000$ & $3.60 \pm 0.40$ & 4.07 & B2 III/IVe & -5.6 & 160 \\
B16 & $10000 \pm 1500$ & $2.60 \pm 0.50$ & 4.02 & B9.5 Ib/II & - & $<100$ \\
B18 & $32000 \pm 1000$ & $4.50 \pm 0.20$ & 4.49 & B0 Ve & -9.7 & 180 \\
B21 & $22000 \pm 1000$ & $3.00 \pm 0.25$ & 4.59 & B1.5 II/IIIe & -36.6 & 320 \\
B22 & $20000 \pm 1000$ & $3.20 \pm 0.20$ & 4.53 & B2 IIe & -1.9 & 150 \\
B24 & $25000 \pm 3000$ & $3.90 \pm 0.50$ & 4.42 & B1 IVe & -1.6 & 150 \\
B28 & $30000 \pm 3000$ & $4.40 \pm 0.40$ & 4.34 & B0 Ve & -0.6 & 125 \\
B30 & $20500 \pm 1000$ & $3.25 \pm 0.25$ & 4.57 & B2 II & - & $<100$ \\
B35 & $25000 \pm 5000$ & $3.50 \pm 0.50$ & 4.38 & B1.5 III/IVe & -49.2 & 400 \\
B37 & $18000 \pm 1000$ & $2.60 \pm 0.20$ & 4.81 & B3 Ib & - & $<100$ \\
\hline
\end{tabular}

discs surrounding the Be stars, as it is normally supposed, then for a given Be star the observed $\mathrm{H}_{\alpha}$ emission should be higher for smaller $\sin i$ values. Since we observe the opposite correlation, we suggest that we are seeing the Be stars in NGC 330 under roughly the same inclination angle, so that the $\mathrm{H}_{\alpha}$ emission correlates directly with $v_{\text {rot }}$.

Two interesting conclusions can therefore be drawn: first, that the rotational axes of all stars in NGC 330 are aligned, which has implications on the process of star formation in the cluster, and, secondly, that the extension of the discs surrounding Be stars depends on the stars' rotational velocities.

\section{References}

Feast, M.W.: 1972, Monthly Notices of the RAS 159,113

Grebel, E.K., Richtler, T., de Boer, K.S.: 1992, Astronomy and Astrophysics 254, L5

Lennon, D.J., Mazzali, P.A., Pasian,F., Bonifacio, P., Castellani, V.: 1993 Space Science Reviews, in press

Robertson, J.W.: 1974, Astronomy and Astrophysics, Supplement Series 15, 261 